Cavendish-HEP-03/26

\title{
$W$ hadroproduction at large transverse momentum beyond next-to-leading order
}

\author{
Nikolaos Kidonakis and Agustín Sabio Vera \\ Cavendish Laboratory \\ University of Cambridge \\ Madingley Road \\ Cambridge CB3 OHE, UK
}

\begin{abstract}
We study the production of $W$ bosons at large transverse momentum in $p \bar{p}$ collisions. We show that the next-to-leading order cross section at large transverse momentum is dominated by threshold soft-gluon corrections. We add next-to-next-to-leading-order softgluon corrections to the exact next-to-leading-order differential cross sections. We find that these higher-order corrections provide modest enhancements to the transverse momentum distribution of the $W$ at the Tevatron, and reduce significantly the dependence on the factorization and renormalization scales.
\end{abstract}




\section{Introduction}

The production of $W$ bosons in hadron colliders is a process of relevance in testing the Standard Model and in estimates of backgrounds to new physics. For example, $W b \bar{b}$ production is the principal background to the associated Higgs boson production $p \bar{p} \rightarrow H(\rightarrow b \bar{b}) W$ at the Tevatron [1.

The calculation of the complete next-to-leading-order (NLO) cross section for $W$ hadroproduction at large transverse momentum was presented in Refs. [2, 3]. The NLO results in [2, 3] showed an enhancement of the differential distributions in transverse momentum $Q_{T}$ of the $W$ boson. The $Q_{T}$ distribution falls rapidly with increasing $Q_{T}$, spanning five orders of magnitude in the $30 \mathrm{GeV}<Q_{T}<190 \mathrm{GeV}$ region at the Tevatron. The NLO corrections also considerably stabilize the dependence of the cross section on the factorization and renormalization scales.

The calculation of hard-scattering cross sections near partonic threshold, such as electroweakboson production at high transverse momentum, involves corrections from the emission of soft gluons from the partons in the process. At each order in perturbation theory one encounters large logarithms that arise from incomplete cancellations near partonic threshold between graphs with real emission and virtual graphs. This is due to the limited phase space available for real gluon emission near partonic threshold. These threshold corrections, calculated in the eikonal approximation, exponentiate as a result of the factorization properties [4, 5, 6] of the cross section. The cross section is factorized into functions that describe gluons collinear to the incoming partons, hard quanta, and noncollinear soft-gluons. The renormalization group properties of these functions result in resummation. Such threshold corrections have by now been successfully resummed for many processes [7].

In this paper we further the study of the resummation of threshold logarithms and the expansion of the resummed cross section at next-to-next-to-leading order (NNLO) for electroweak boson hadroproduction, which was first presented in Ref. [8] at next-to-next-to-leading logarithmic (NNLL) accuracy. In Ref. 8] no numerical phenomenological studies were made. In this paper we study the significance of NNLO soft-gluon corrections for $W$-boson production at large transverse momentum. Furthermore we increase the accuracy of the theoretical calculation to next-to-next-to-next-to-leading logarithms (NNNLL). Related studies for direct photon production, including numerical results, were made in Refs. 9, 10]. The partonic subprocesses involved in direct photon production are similar to the ones discussed in this paper.

Recently, a unified approach to the calculation of NNLO soft and virtual corrections for processes in hadron-hadron and lepton-hadron colliders has been presented in Ref. [11. This work unifies and extends previous approaches by going beyond NNLL accuracy. We follow this reference and calculate the NNLO soft corrections to $W$ production at large transverse momentum at the Tevatron. The form of our theoretical results differs slightly from (but is consistent with) the one in Ref. [8], partly in that here we use the transverse momentum $Q_{T}$ as the hard scale instead of the mass of the $W$.

We note that there has also been work on resummation of Sudakov logarithms at small transverse momentum for electroweak boson production [12, 13, as well as on joint resummation of $Q_{T}$ and threshold logarithms 14 .

In Section 2 we discuss some of the kinematics of the partonic subprocesses involved and we define the corrections to be calculated. In Section 3 we provide results for the NLO soft and 
virtual, and the NNLO soft-gluon corrections. The NLO soft corrections agree near partonic threshold with the exact NLO calculations in Refs. 2, 3] while our NNLO soft-gluon corrections provide new predictions. In Section 4 we study the numerical effect of the NNLO soft-gluon corrections to $W$ hadroproduction at the Tevatron Run I and II, while also showing that the NLO cross section is dominated by soft-gluon corrections.

\section{Kinematics and plus distributions}

For the hadronic production of a $W$ boson, with mass $m_{W}$,

$$
h_{A}\left(P_{A}\right)+h_{B}\left(P_{B}\right) \longrightarrow W(Q)+X,
$$

we can write the factorized single-particle-inclusive cross section as

$$
\begin{aligned}
& E_{Q} \frac{d \sigma_{h_{A} h_{B} \rightarrow W(Q)+X}}{d^{3} Q}=\sum_{f} \int d x_{1} d x_{2} \phi_{f_{a} / h_{A}}\left(x_{1}, \mu_{F}^{2}\right) \phi_{f_{b} / h_{B}}\left(x_{2}, \mu_{F}^{2}\right) \\
& \times E_{Q} \frac{d \hat{\sigma}_{f_{a} f_{b} \rightarrow W(Q)+X}}{d^{3} Q}\left(s, t, u, Q, \mu_{F}, \alpha_{s}\left(\mu_{R}^{2}\right)\right)
\end{aligned}
$$

where $E_{Q}=Q^{0}, \phi_{f / h}$ is the parton distribution for parton $f$ in hadron $h$, and $\hat{\sigma}$ is the perturbative parton-level cross section. The initial-state collinear singularities are factorized into the parton distributions at factorization scale $\mu_{F}$, while $\mu_{R}$ is the renormalization scale.

At the parton level, the lowest-order subprocesses for the production of a $W$ boson and a parton are

$$
\begin{aligned}
& q\left(p_{a}\right)+g\left(p_{b}\right) \longrightarrow W(Q)+q\left(p_{c}\right), \\
& q\left(p_{a}\right)+\bar{q}\left(p_{b}\right) \longrightarrow W(Q)+g\left(p_{c}\right) .
\end{aligned}
$$

The hadronic and partonic kinematical invariants in the process are

$$
\begin{aligned}
& S=\left(P_{A}+P_{B}\right)^{2}, T=\left(P_{A}-Q\right)^{2}, U=\left(P_{B}-Q\right)^{2}, S_{2}=S+T+U-Q^{2}, \\
& s=\left(p_{a}+p_{b}\right)^{2}, t=\left(p_{a}-Q\right)^{2}, u=\left(p_{b}-Q\right)^{2}, s_{2}=s+t+u-Q^{2},
\end{aligned}
$$

where $S_{2}$ and $s_{2}$ are the invariant masses of the system recoiling against the electroweak boson at the hadron and parton levels, respectively. $s_{2}=\left(p_{a}+p_{b}-Q\right)^{2}$ parametrizes the inelasticity of the parton scattering, taking the value $s_{2}=0$ for one-parton production. Since $x_{i}$ is the initialparton momentum fraction, defined by $p_{a}=x_{1} P_{A}$ and $p_{b}=x_{2} P_{B}$, the hadronic and partonic kinematical invariants are related by $s=x_{1} x_{2} S, t-Q^{2}=x_{1}\left(T-Q^{2}\right)$, and $u-Q^{2}=x_{2}\left(U-Q^{2}\right)$.

In general, the partonic cross section $\hat{\sigma}$ includes distributions with respect to $s_{2}$ at $n$-th order in the strong coupling $\alpha_{s}$ of the type

$$
\left[\frac{\ln ^{m}\left(s_{2} / Q_{T}^{2}\right)}{s_{2}}\right]_{+}, \quad m \leq 2 n-1,
$$


defined by their integral with any smooth function $f$ by

$$
\begin{aligned}
\int_{0}^{s_{2 \max }} d s_{2} f\left(s_{2}\right)\left[\frac{\ln ^{m}\left(s_{2} / Q_{T}^{2}\right)}{s_{2}}\right]_{+} \equiv & \int_{0}^{s_{2} \max } d s_{2} \frac{\ln ^{m}\left(s_{2} / Q_{T}^{2}\right)}{s_{2}}\left[f\left(s_{2}\right)-f(0)\right] \\
& +\frac{1}{m+1} \ln ^{m+1}\left(\frac{s_{2 \max }}{Q_{T}^{2}}\right) f(0) .
\end{aligned}
$$

These "plus" distributions are the remnants of cancellations between real and virtual contributions to the cross section. Note that in Ref. [8] we used $Q$ instead of $Q_{T}$ in the plus distributions. Here we prefer to use $Q_{T}$ as we find it a slightly better physical hard scale for the $Q_{T}$ distributions that we will be calculating. Below we will make use of the terminology that at $n$-th order in $\alpha_{s}$ the leading logarithms (LL) are those with $m=2 n-1$ in Eq. (2.5), next-to-leading logarithms (NLL) with $m=2 n-2$, next-to-next-to-leading logarithms (NNLL) with $m=2 n-3$, and next-to-next-to-next-to-leading logarithms (NNNLL) with $m=2 n-4$.

\section{Next-to-next-to-leading order soft-gluon corrections}

In this section, for each of the subprocesses in Eq. (2.3) we first present the NLO soft and virtual corrections. We then present the NNLO soft-gluon corrections. We work in the $\overline{\mathrm{MS}}$ scheme throughout.

\subsection{The $q g \longrightarrow W q$ subprocess}

The Born differential cross section for this process is

$$
E_{Q} \frac{d \sigma_{q g \rightarrow W q}^{B}}{d^{3} Q}=F_{q g \rightarrow W q}^{B} \delta\left(s_{2}\right)
$$

where

$$
\begin{aligned}
F_{q g \rightarrow W q}^{B} & =\frac{\alpha \alpha_{s}\left(\mu_{R}^{2}\right) C_{F}}{s\left(N_{c}^{2}-1\right)} A^{q g} \sum_{f}\left|L_{f f_{a}}\right|^{2}, \\
A^{q g} & =-\left(\frac{s}{t}+\frac{t}{s}+\frac{2 u Q^{2}}{s t}\right)
\end{aligned}
$$

with $L$ the left-handed couplings of the $W$ boson to the quark line, $f$ the quark flavor and $\sum_{f}$ the sum over the flavors allowed by the CKM mixing and by the energy threshold. For the $L$ couplings we choose the conventions of Ref. [3]. Also $C_{F}=\left(N_{c}^{2}-1\right) /\left(2 N_{c}\right)$ with $N_{c}=3$ the number of colors.

We can write the NLO soft and virtual corrections for $q g \longrightarrow W q$ in single-particle inclusive kinematics as

$$
E_{Q} \frac{d \hat{\sigma}_{q g \rightarrow W q}^{(1)}}{d^{3} Q}=F_{q g \rightarrow W q}^{B} \frac{\alpha_{s}\left(\mu_{R}^{2}\right)}{\pi}\left\{c_{3}^{q g}\left[\frac{\ln \left(s_{2} / Q_{T}^{2}\right)}{s_{2}}\right]_{+}+c_{2}^{q g}\left[\frac{1}{s_{2}}\right]_{+}+c_{1}^{q g} \delta\left(s_{2}\right)\right\} .
$$


Note that the $\left[\ln \left(s_{2} / Q_{T}^{2}\right) / s_{2}\right]+$ term (which is the LL, since $m=n=1$ in Eq. (2.5)) and the $\left[1 / s_{2}\right]_{+}$term (NLL, since $\left.n=1, m=0\right)$ are the soft gluon corrections. The $\delta\left(s_{2}\right)$ term is the contribution from the virtual corrections. Below we use the terminology "NLO-NLL" to indicate when, at next-to-leading order, we include the LL and NLL soft-gluon terms (as well as scale dependent terms in $\left.\delta\left(s_{2}\right)\right)$. Also the terminology "soft and virtual" is used to denote all the terms in Eq. (3.3). As we will see, we need to know both soft and virtual corrections at NLO in order to derive the soft terms at NNLO with at least NNLL accuracy.

The NLO coefficients in Eq. (3.3) are $c_{3}^{q g}=C_{F}+2 C_{A}$,

$$
c_{2}^{q g}=-\left(C_{F}+C_{A}\right) \ln \left(\frac{\mu_{F}^{2}}{Q_{T}^{2}}\right)-\frac{3}{4} C_{F}-C_{A} \ln \left(\frac{t u}{s Q_{T}^{2}}\right),
$$

and

$$
c_{1}^{q g}=\frac{1}{2 A^{q g}}\left[B_{1}^{q g}+B_{2}^{q g} n_{f}+C_{1}^{q g}+C_{2}^{q g} n_{f}\right]+\frac{c_{3}^{q g}}{2} \ln ^{2}\left(\frac{Q_{T}^{2}}{Q^{2}}\right)+c_{2}^{q g} \ln \left(\frac{Q_{T}^{2}}{Q^{2}}\right),
$$

with $C_{A}=N_{c}, n_{f}=5$ the number of light quark flavors, and $B_{1}^{q g}, B_{2}^{q g}, C_{1}^{q g}$, and $C_{2}^{q g}$ as given in the Appendix of Ref. [3] but without the renormalization counterterms and using $f_{A} \equiv \ln \left(A / Q^{2}\right)=0$.

Note that we can write $c_{2}^{q g} \equiv c_{2 \mu}^{q g}+T_{2}^{q g}$ with $c_{2 \mu}^{q g} \equiv-\left(C_{F}+C_{A}\right) \ln \left(\mu_{F}^{2} / s\right)$. Similarly we also write $c_{1}^{q g} \equiv c_{1 \mu}^{q g}+T_{1}^{q g}$ with

$$
c_{1 \mu}^{q g} \equiv \ln \left(\frac{\mu_{F}^{2}}{s}\right)\left[-\frac{\beta_{0}}{4}+C_{F}\left(\ln \left(\frac{-u}{Q_{T}^{2}}\right)-\frac{3}{4}\right)+C_{A} \ln \left(\frac{-t}{Q_{T}^{2}}\right)\right]+\frac{\beta_{0}}{4} \ln \left(\frac{\mu_{R}^{2}}{s}\right),
$$

where $\beta_{0}=\left(11 C_{A}-2 n_{f}\right) / 3$ is the lowest-order beta function. Thus, $c_{2 \mu}^{q g}$ and $c_{1 \mu}^{q g}$ are scaledependent parts of the $c_{2}^{q g}$ and $c_{1}^{q g}$ coefficients, respectively, while $T_{2}^{q g}$ and $T_{1}^{q g}$ are scaleindependent parts. We have kept the factorization scale $\mu_{F}$ and the renormalization scale $\mu_{R}$ separate. Finally, another useful notation is $c_{1}^{q g} \equiv c_{1 \mu}^{\prime q g}+T_{1}^{\prime q g}$ where $c_{1 \mu}^{\prime q g}$ is defined as $c_{1 \mu}^{q g}$ with $Q_{T}^{2}$ instead of $s$ in the denominators of the logarithms involving the scales $\mu_{F}$ and $\mu_{R}$.

Using the above conventions, the NNLO soft and virtual corrections for $q g \longrightarrow W q$ can be written as

$$
E_{Q} \frac{d \hat{\sigma}_{q g \rightarrow W q}^{(2)}}{d^{3} Q}=F_{q g \rightarrow W q}^{B} \frac{\alpha_{s}^{2}\left(\mu_{R}^{2}\right)}{\pi^{2}}{\hat{\sigma^{\prime}}}_{q g \rightarrow W q}^{(2)}
$$

with

$$
\begin{gathered}
{\hat{\sigma^{\prime}}}_{q g \rightarrow W q}^{(2)}=\frac{1}{2}\left(c_{3}^{q g}\right)^{2}\left[\frac{\ln ^{3}\left(s_{2} / Q_{T}^{2}\right)}{s_{2}}\right]_{+}+\left[\frac{3}{2} c_{3}^{q g} c_{2}^{q g}-\frac{\beta_{0}}{4} c_{3}^{q g}+C_{F} \frac{\beta_{0}}{8}\right]\left[\frac{\ln ^{2}\left(s_{2} / Q_{T}^{2}\right)}{s_{2}}\right]_{+} \\
+\left\{c_{3}^{q g} c_{1}^{q g}+\left(c_{2}^{q g}\right)^{2}-\zeta_{2}\left(c_{3}^{q g}\right)^{2}-\frac{\beta_{0}}{2} T_{2}^{q g}+\frac{\beta_{0}}{4} c_{3}^{q g} \ln \left(\frac{\mu_{R}^{2}}{s}\right)+\left(C_{F}+C_{A}\right) K\right. \\
\left.+C_{F}\left[-\frac{K}{2}+\frac{\beta_{0}}{4} \ln \left(\frac{Q_{T}^{2}}{s}\right)\right]-\frac{3}{16} \beta_{0} C_{F}\right\}\left[\frac{\ln \left(s_{2} / Q_{T}^{2}\right)}{s_{2}}\right]_{+} \\
+\left\{c_{2}^{q g} c_{1}^{q g}-\zeta_{2} c_{2}^{q g} c_{3}^{q g}+\zeta_{3}\left(c_{3}^{q g}\right)^{2}-\frac{\beta_{0}}{2} T_{1}^{q g}+\frac{\beta_{0}}{4} c_{2}^{q g} \ln \left(\frac{\mu_{R}^{2}}{s}\right)+\mathcal{G}_{q g}^{(2)}\right.
\end{gathered}
$$




$$
\begin{aligned}
&+\left(C_{F}+C_{A}\right)\left[\frac{\beta_{0}}{8} \ln ^{2}\left(\frac{\mu_{F}^{2}}{s}\right)-\frac{K}{2} \ln \left(\frac{\mu_{F}^{2}}{s}\right)\right]-C_{F} K \ln \left(\frac{-u}{Q_{T}^{2}}\right)-C_{A} K \ln \left(\frac{-t}{Q_{T}^{2}}\right) \\
&\left.+C_{F}\left[\frac{\beta_{0}}{8} \ln ^{2}\left(\frac{Q_{T}^{2}}{s}\right)-\frac{K}{2} \ln \left(\frac{Q_{T}^{2}}{s}\right)\right]-\frac{3}{16} \beta_{0} C_{F} \ln \left(\frac{Q_{T}^{2}}{s}\right)\right\}\left[\frac{1}{s_{2}}\right]_{+} \\
&+\left\{\frac{1}{2}\left(c_{1 \mu}^{\prime q g}\right)^{2}+c_{1 \mu}^{\prime q g} T_{1}^{\prime q g}+\frac{\beta_{0}}{4} c_{1 \mu}^{\prime q g} \ln \left(\frac{Q_{T}^{2}}{s}\right)+\frac{\beta_{0}}{4} c_{1}^{q g} \ln \left(\frac{\mu_{R}^{2}}{Q_{T}^{2}}\right)-\left(C_{F}+C_{A}\right)^{2} \frac{\zeta_{2}}{2} \ln ^{2}\left(\frac{\mu_{F}^{2}}{Q_{T}^{2}}\right)\right. \\
&+\left(C_{F}+C_{A}\right) \ln \left(\frac{\mu_{F}^{2}}{Q_{T}^{2}}\right)\left(\zeta_{2} T_{2}^{q g}-\zeta_{2}\left(C_{F}+C_{A}\right) \ln \left(\frac{Q_{T}^{2}}{s}\right)-\zeta_{3} c_{3}^{q g}\right) \\
&+\frac{\beta_{0}^{2}}{32} \ln { }^{2}\left(\frac{\mu_{R}^{2}}{Q_{T}^{2}}\right)-\frac{\beta_{0}^{2}}{16} \ln \left(\frac{\mu_{R}^{2}}{Q_{T}^{2}}\right) \ln \left(\frac{Q_{T}^{2}}{s}\right)+\frac{\beta_{1}}{16} \ln \left(\frac{\mu_{R}^{2}}{Q_{T}^{2}}\right) \\
&+\frac{\beta_{0}}{8}\left[\frac{3}{4} C_{F}+\frac{\beta_{0}}{4}-C_{F} \ln \left(\frac{-u}{Q_{T}^{2}}\right)-C_{A} \ln \left(\frac{-t}{Q_{T}^{2}}\right)\right]\left[\ln ^{2}\left(\frac{\mu_{F}^{2}}{Q_{T}^{2}}\right)+2 \ln \left(\frac{\mu_{F}^{2}}{Q_{T}^{2}}\right) \ln \left(\frac{Q_{T}^{2}}{s}\right)\right] \\
&+C_{F} \frac{K}{2} \ln \left(\frac{-u}{Q_{T}^{2}}\right) \ln \left(\frac{\mu_{F}^{2}}{Q_{T}^{2}}\right)+C_{A} \frac{K}{2} \ln \left(\frac{-t}{Q_{T}^{2}}\right) \ln \left(\frac{\mu_{F}^{2}}{Q_{T}^{2}}\right)-\left(\gamma_{q / q}^{\prime(2)}+\gamma_{g / g}^{\prime(2)}\right) \ln \left(\frac{\mu_{F}^{2}}{Q_{T}^{2}}\right) \\
&\left.+R_{q g}^{(2)}\right\} \delta\left(s_{2}\right),
\end{aligned}
$$

where $K=C_{A}\left(67 / 18-\zeta_{2}\right)-5 n_{f} / 9$ is a two-loop function in the $\overline{\mathrm{MS}}$ scheme [15], $\zeta_{2}=\pi^{2} / 6$ and $\zeta_{3}=1.2020569 \cdots$ are Riemann zeta functions, $\beta_{1}=34 C_{A}^{2} / 3-2 n_{f}\left(C_{F}+5 C_{A} / 3\right)$ is the next-to-leading order beta function, and

$$
\begin{gathered}
\gamma_{q / q}^{\prime(2)}=C_{F}^{2}\left(\frac{3}{32}-\frac{3}{4} \zeta_{2}+\frac{3}{2} \zeta_{3}\right)+C_{F} C_{A}\left(-\frac{3}{4} \zeta_{3}+\frac{11}{12} \zeta_{2}+\frac{17}{96}\right)+n_{f} C_{F}\left(-\frac{\zeta_{2}}{6}-\frac{1}{48}\right), \\
\gamma_{g / g}^{\prime(2)}=C_{A}^{2}\left(\frac{2}{3}+\frac{3}{4} \zeta_{3}\right)-n_{f}\left(\frac{C_{F}}{8}+\frac{C_{A}}{6}\right),
\end{gathered}
$$

are two-loop parton anomalous dimensions [16, 17].

The soft corrections are the $\left[\ln ^{3}\left(s_{2} / Q_{T}^{2}\right) / s_{2}\right]_{+}$term (which is the LL, since $n=2, m=3$ in Eq. (2.5) $),\left[\ln ^{2}\left(s_{2} / Q_{T}^{2}\right) / s_{2}\right]+\operatorname{term}(\mathrm{NLL}, n=2, m=2),\left[\ln \left(s_{2} / Q_{T}^{2}\right) / s_{2}\right]_{+}$term (NNLL, $n=2$, $m=1$ ), and $\left[1 / s_{2}\right]_{+}$term (NNNLL, $n=2, m=0$ ). The function $\mathcal{G}_{q g}^{(2)}$ in the NNNLL term denotes a set of two-loop contributions [10, 11] and is given by

$$
\begin{aligned}
\mathcal{G}_{q g}^{(2)}= & C_{F}^{2}\left(-\frac{3}{32}+\frac{3}{4} \zeta_{2}-\frac{3}{2} \zeta_{3}\right)+C_{F} C_{A}\left(\frac{3}{4} \zeta_{3}-\frac{11}{12} \zeta_{2}-\frac{189}{32}\right) \\
& +C_{A}^{2}\left(\frac{7}{4} \zeta_{3}+\frac{11}{3} \zeta_{2}-\frac{41}{216}\right)+n_{f} C_{F}\left(\frac{1}{6} \zeta_{2}+\frac{17}{16}\right)+n_{f} C_{A}\left(-\frac{2}{3} \zeta_{2}-\frac{5}{108}\right) .
\end{aligned}
$$

Note that we have not included in $\mathcal{G}_{q g}^{(2)}$ two-loop process-dependent contributions; however from related studies for other processes, including top hadroproduction [18, and direct photon production [10] we expect such contributions to be small. It is actually the $-\zeta_{2} c_{2}^{q g} c_{3}^{q g}+\zeta_{3}\left(c_{3}^{q g}\right)^{2}$ terms in Eq. (3.8) that provide the major contribution to the NNNLL term.

We use the terminology "NNLO-NNLL" below, to indicate that we include the LL, NLL, and NNLL terms at NNLO, while we use "NNLO-NNNLL" to indicate that in addition we include the NNNLL terms as well. 
The term proportional to $\delta\left(s_{2}\right)$ includes the virtual corrections. We remind the reader that here we do not calculate the full virtual corrections; the term $R_{q g}^{(2)}$ that denotes the scaleindependent virtual corrections is currently unknown. However, we have calculated explicitly here all the $\delta\left(s_{2}\right)$ terms that include the scale-dependence. Note that when discussing scale dependence at NNLO-NNNLL we include all scale-dependent $\delta\left(s_{2}\right)$ terms, which is a consistent approach from the resummation procedure, see Ref. [19].

\subsection{The $q \bar{q} \longrightarrow W g$ subprocess}

Next, we consider the $q \bar{q} \longrightarrow W g$ partonic subprocess. Here the Born differential cross section is

$$
E_{Q} \frac{d \sigma_{q \bar{q} \rightarrow W g}^{B}}{d^{3} Q}=F_{q \bar{q} \rightarrow W g}^{B} \delta\left(s_{2}\right),
$$

where

$$
\begin{aligned}
F_{q \bar{q} \rightarrow W g}^{B} & =\frac{\alpha \alpha_{s}\left(\mu_{R}^{2}\right) C_{F}}{s N_{c}} A^{q \bar{q}}\left|L_{f_{b} f_{a}}\right|^{2}, \\
A^{q \bar{q}} & =\frac{u}{t}+\frac{t}{u}+\frac{2 Q^{2} s}{t u} .
\end{aligned}
$$

The NLO soft and virtual corrections in single-particle inclusive kinematics can be written as

$$
E_{Q} \frac{d \hat{\sigma}_{q \bar{q} \rightarrow W g}^{(1)}}{d^{3} Q}=F_{q \bar{q} \rightarrow W g}^{B} \frac{\alpha_{s}\left(\mu_{R}^{2}\right)}{\pi}\left\{c_{3}^{q \bar{q}}\left[\frac{\ln \left(s_{2} / Q_{T}^{2}\right)}{s_{2}}\right]_{+}+c_{2}^{q \bar{q}}\left[\frac{1}{s_{2}}\right]_{+}+c_{1}^{q \bar{q}} \delta\left(s_{2}\right)\right\} .
$$

Here the NLO coefficients are $c_{3}^{q \bar{q}}=4 C_{F}-C_{A}$,

$$
c_{2}^{q \bar{q}}=-2 C_{F} \ln \left(\frac{\mu_{F}^{2}}{Q_{T}^{2}}\right)-\left(2 C_{F}-C_{A}\right) \ln \left(\frac{t u}{s Q_{T}^{2}}\right)-\frac{\beta_{0}}{4}
$$

and

$$
c_{1}^{q \bar{q}}=\frac{1}{2 A^{q \bar{q}}}\left[B_{1}^{q \bar{q}}+C_{1}^{q \bar{q}}+\left(B_{2}^{q \bar{q}}+D_{a a}^{(0)}\right) n_{f}\right]+\frac{c_{3}^{q \bar{q}}}{2} \ln ^{2}\left(\frac{Q_{T}^{2}}{Q^{2}}\right)+c_{2}^{q \bar{q}} \ln \left(\frac{Q_{T}^{2}}{Q^{2}}\right),
$$

with $B_{1}^{q \bar{q}}, B_{2}^{q \bar{q}}, C_{1}^{q \bar{q}}$, and $D_{a a}^{(0)}$ as given in the Appendix of Ref. 3] but without the renormalization counterterms and using $f_{A}=0$. Again, we can write $c_{1}^{q \bar{q}}=c_{1 \mu}^{q \bar{q}}+T_{1}^{q \bar{q}}$ with

$$
c_{1 \mu}^{q \bar{q}}=\ln \left(\frac{\mu_{F}^{2}}{s}\right) C_{F}\left[\ln \left(\frac{t u}{Q_{T}^{4}}\right)-\frac{3}{2}\right]+\frac{\beta_{0}}{4} \ln \left(\frac{\mu_{R}^{2}}{s}\right),
$$

and $c_{2}^{q \bar{q}}=c_{2 \mu}^{q \bar{q}}+T_{2}^{q \bar{q}}$ with $c_{2 \mu}^{q \bar{q}}=-2 C_{F} \ln \left(\mu_{F}^{2} / s\right)$. Finally, another useful notation is $c_{1}^{q \bar{q}} \equiv$ $c_{1 \mu}^{\prime q \bar{q}}+T_{1}^{\prime q \bar{q}}$ where $c_{1 \mu}^{\prime q \bar{q}}$ is defined as $c_{1 \mu}^{q \bar{q}}$ with $Q_{T}^{2}$ instead of $s$ in the denominators of the logarithms involving the scales $\mu_{F}$ and $\mu_{R}$. 
The NNLO soft and virtual corrections for $q \bar{q} \longrightarrow W g$ can be written as

$$
E_{Q} \frac{d \hat{\sigma}_{q \bar{q} \rightarrow W g}^{(2)}}{d^{3} Q}=F_{q \bar{q} \rightarrow W g}^{B} \frac{\alpha_{s}^{2}\left(\mu_{R}^{2}\right)}{\pi^{2}} \hat{\sigma}_{q \bar{q} \rightarrow W g}^{(2)}
$$

with

$$
\begin{aligned}
& {\hat{\sigma^{\prime}}}_{q \bar{q} \rightarrow W g}^{(2)}=\frac{1}{2}\left(c_{3}^{q \bar{q}}\right)^{2}\left[\frac{\ln ^{3}\left(s_{2} / Q_{T}^{2}\right)}{s_{2}}\right]_{+}+\left[\frac{3}{2} c_{3}^{q \bar{q}} c_{2}^{q \bar{q}}-\frac{\beta_{0}}{4} c_{3}^{q \bar{q}}+C_{A} \frac{\beta_{0}}{8}\right]\left[\frac{\ln ^{2}\left(s_{2} / Q_{T}^{2}\right)}{s_{2}}\right]_{+} \\
& +\left\{c_{3}^{q \bar{q}} c_{1}^{q \bar{q}}+\left(c_{2}^{q \bar{q}}\right)^{2}-\zeta_{2}\left(c_{3}^{q \bar{q}}\right)^{2}-\frac{\beta_{0}}{2} T_{2}^{q \bar{q}}+\frac{\beta_{0}}{4} c_{3}^{q \bar{q}} \ln \left(\frac{\mu_{R}^{2}}{s}\right)+2 C_{F} K\right. \\
& \left.+C_{A}\left[-\frac{K}{2}+\frac{\beta_{0}}{4} \ln \left(\frac{Q_{T}^{2}}{s}\right)\right]-\frac{\beta_{0}^{2}}{16}\right\}\left[\frac{\ln \left(s_{2} / Q_{T}^{2}\right)}{s_{2}}\right]_{+} \\
& +\left\{c_{2}^{q \bar{q}} c_{1}^{q \bar{q}}-\zeta_{2} c_{2}^{q \bar{q}} c_{3}^{q \bar{q}}+\zeta_{3}\left(c_{3}^{q \bar{q}}\right)^{2}-\frac{\beta_{0}}{2} T_{1}^{q \bar{q}}+\frac{\beta_{0}}{4} c_{2}^{q \bar{q}} \ln \left(\frac{\mu_{R}^{2}}{s}\right)+\mathcal{G}_{q \bar{q}}^{(2)}\right. \\
& +C_{F}\left[\frac{\beta_{0}}{4} \ln ^{2}\left(\frac{\mu_{F}^{2}}{s}\right)-K \ln \left(\frac{\mu_{F}^{2}}{s}\right)-K \ln \left(\frac{t u}{Q_{T}^{4}}\right)\right] \\
& \left.+C_{A}\left[\frac{\beta_{0}}{8} \ln ^{2}\left(\frac{Q_{T}^{2}}{s}\right)-\frac{K}{2} \ln \left(\frac{Q_{T}^{2}}{s}\right)\right]-\frac{\beta_{0}^{2}}{16} \ln \left(\frac{Q_{T}^{2}}{s}\right)\right\}\left[\frac{1}{s_{2}}\right]_{+} \\
& +\left\{\frac{1}{2}\left(c_{1 \mu}^{\prime q \bar{q}}\right)^{2}+c_{1 \mu}^{\prime q \bar{q}} T_{1}^{\prime q \bar{q}}+\frac{\beta_{0}}{4} c_{1 \mu}^{\prime q \bar{q}} \ln \left(\frac{Q_{T}^{2}}{s}\right)+\frac{\beta_{0}}{4} c_{1}^{q \bar{q}} \ln \left(\frac{\mu_{R}^{2}}{Q_{T}^{2}}\right)\right. \\
& -2 C_{F}^{2} \zeta_{2} \ln ^{2}\left(\frac{\mu_{F}^{2}}{Q_{T}^{2}}\right)+2 C_{F} \ln \left(\frac{\mu_{F}^{2}}{Q_{T}^{2}}\right)\left(\zeta_{2} T_{2}^{q \bar{q}}-2 \zeta_{2} C_{F} \ln \left(\frac{Q_{T}^{2}}{s}\right)-\zeta_{3} c_{3}^{q \bar{q}}\right) \\
& -\frac{\beta_{0}^{2}}{32} \ln ^{2}\left(\frac{\mu_{R}^{2}}{Q_{T}^{2}}\right)-\frac{\beta_{0}^{2}}{16} \ln \left(\frac{\mu_{R}^{2}}{Q_{T}^{2}}\right) \ln \left(\frac{Q_{T}^{2}}{s}\right)+\frac{\beta_{1}}{16} \ln \left(\frac{\mu_{R}^{2}}{Q_{T}^{2}}\right) \\
& +\frac{\beta_{0}}{8}\left[\frac{3}{2} C_{F}-C_{F} \ln \left(\frac{t u}{Q_{T}^{4}}\right)\right]\left[\ln ^{2}\left(\frac{\mu_{F}^{2}}{Q_{T}^{2}}\right)+2 \ln \left(\frac{\mu_{F}^{2}}{Q_{T}^{2}}\right) \ln \left(\frac{Q_{T}^{2}}{s}\right)\right] \\
& \left.+C_{F} \frac{K}{2} \ln \left(\frac{t u}{Q_{T}^{4}}\right) \ln \left(\frac{\mu_{F}^{2}}{Q_{T}^{2}}\right)-2{\gamma^{\prime}}_{q / q}^{(2)} \ln \left(\frac{\mu_{F}^{2}}{Q_{T}^{2}}\right)+R_{q \bar{q}}^{(2)}\right\} \delta\left(s_{2}\right),
\end{aligned}
$$

The function $\mathcal{G}_{q \bar{q}}^{(2)}$ denotes again a set of two-loop contributions [10, 11] and is given by

$$
\mathcal{G}_{q \bar{q}}^{(2)}=C_{F} C_{A}\left(\frac{7}{2} \zeta_{3}+\frac{22}{3} \zeta_{2}-\frac{299}{27}\right)+n_{f} C_{F}\left(-\frac{4}{3} \zeta_{2}+\frac{50}{27}\right) .
$$

Again, we have not included in $\mathcal{G}_{q \bar{q}}^{(2)}$ two-loop process-dependent contributions.

We also note that we do not calculate the full virtual corrections. All $\delta\left(s_{2}\right)$ terms shown here only include the scale-dependence and are used in the study of the scale dependence of the NNLO-NNNLL cross section. The term $R_{q \bar{q}}^{(2)}$ is unknown.

\section{Numerical results}

For the $W$ hadroproduction cross section for $h_{A}\left(P_{A}\right)+h_{B}\left(P_{B}\right) \rightarrow W(Q)+X$ we defined the hadronic and partonic kinematical invariants in Section 2. The hadronic kinematical variables 
$T$ and $U$ can be written alternatively as $T=m_{W}^{2}-m_{T} \sqrt{S} e^{-y}$ and $U=m_{W}^{2}-m_{T} \sqrt{S} e^{y}$. Here $m_{W}$ is the $W$ mass, $m_{T}=\sqrt{Q_{T}^{2}+m_{W}^{2}}$ is the transverse mass, and $y$ is the rapidity of the vector boson. The differential $Q_{T}$ distribution can then be written as

$$
\begin{aligned}
\frac{d \sigma_{h_{A} h_{B} \rightarrow W+X}}{d Q_{T}^{2}}\left(S, m_{W}^{2}, Q_{T}\right) & =\sum_{f} \int_{0}^{1} d y^{\prime} \int_{A}^{1} d x_{1} \int_{0}^{s_{2}^{\max }} d s_{2} \frac{2 \pi Y}{x_{1} S-\sqrt{S} m_{T} e^{y}} \\
& \times \phi_{f_{a} / h_{A}}\left(x_{1}, \mu_{F}^{2}\right) \phi_{f_{b} / h_{B}}\left(x_{2}, \mu_{F}^{2}\right) E_{Q} \frac{d \hat{\sigma}_{f_{a} f_{b} \rightarrow W+X}}{d^{3} Q}\left(x_{1}, x_{2}, y\right),
\end{aligned}
$$

where $Y=\ln \left(B+\sqrt{B^{2}-1}\right), B=\left(S+m_{W}^{2}\right) /\left(2 m_{T} \sqrt{S}\right), y=Y\left(2 y^{\prime}-1\right)$,

$$
\begin{aligned}
s_{2}^{\max } & =m_{W}^{2}-\sqrt{S} m_{T} e^{y}+x_{1}\left(S-\sqrt{S} m_{T} e^{-y}\right), \\
A & =\frac{\sqrt{S} m_{T} e^{y}-m_{W}^{2}}{S-\sqrt{S} m_{T} e^{-y}}, \quad x_{2}=\frac{s_{2}-m_{W}^{2}+\sqrt{S} m_{T} x_{1} e^{-y}}{x_{1} S-\sqrt{S} m_{T} e^{y}} .
\end{aligned}
$$

We now apply our results to $W$ hadroproduction at large transverse momentum at the Tevatron. Throughout we use the MRST2002 approximate NNLO parton densities [20]. In Fig. 1 we plot the transverse momentum distribution, $d \sigma / d Q_{T}^{2}$, for $W$ hadroproduction at the Tevatron Run I with $\sqrt{S}=1.8 \mathrm{TeV}$. Here we have set $\mu_{F}=\mu_{R}=Q_{T}$. We plot Born, exact NLO [2, 3], NNLO-NNLL, and NNLO-NNNLL results. We see that the NLO corrections provide a significant enhancement of the Born cross section. The NNLO-NNLL corrections provide a further modest enhancement of the $Q_{T}$ distribution. If we increase the accuracy by including the NNNLL contributions, which are negative, then we find that the NNLO-NNNLL cross section lies between the NLO and NNLO-NNLL results. Since it is hard to distinguish between the curves in Fig. 1, we provide another figure, Fig. 2, which emphasizes the high- $Q_{T}$ region where the soft-gluon approximation holds best.

In Fig. 3 we plot the $K$-factors, i.e. the ratios of cross sections at various orders and accuracies to the Born cross section, all with $\mu_{F}=\mu_{R}=Q_{T}$, in the high- $Q_{T}$ region. We also show the ratio of the exact NLO to the NLO-NLL cross section. It is clear from this line being very close to 1 that the NLO-NLL result is a very good approximation to the full NLO result, i.e. the soft-gluon corrections overwhelmingly dominate the NLO cross section. The difference between NLO and NLO-NLL is only $2 \%$ for $Q_{T}>90 \mathrm{GeV}$ and less than $10 \%$ for lower $Q_{T}$ down to $30 \mathrm{GeV}$. The fact that the soft-gluon corrections dominate the NLO cross section is a major justification for studying the NNLO soft gluon corrections to this process. We can also see that the various $K$-factors shown in Fig. 3 are moderate, and nearly constant over the $Q_{T}$ range shown even though the distributions themselves span two orders of magnitude in this range. The NLO corrections are nearly $30 \%$ over the Born, with the NNLO corrections giving an additional increase, so that the NNLO-NNLL/Born $K$-factor is around 1.4 and the NNLO-NNNLL/Born $K$-factor is around 1.35.

In Fig. 4 we plot the scale dependence of the differential cross section for $Q_{T}=80 \mathrm{GeV}$. We define $\mu \equiv \mu_{F}=\mu_{R}$ and plot the differential cross section versus $\mu / Q_{T}$ over two orders of magnitude: $0.1<\mu / Q_{T}<10$. We note the good stabilization of the cross section when the NLO corrections are included, and the further improvement when the NNLO-NNNLL 


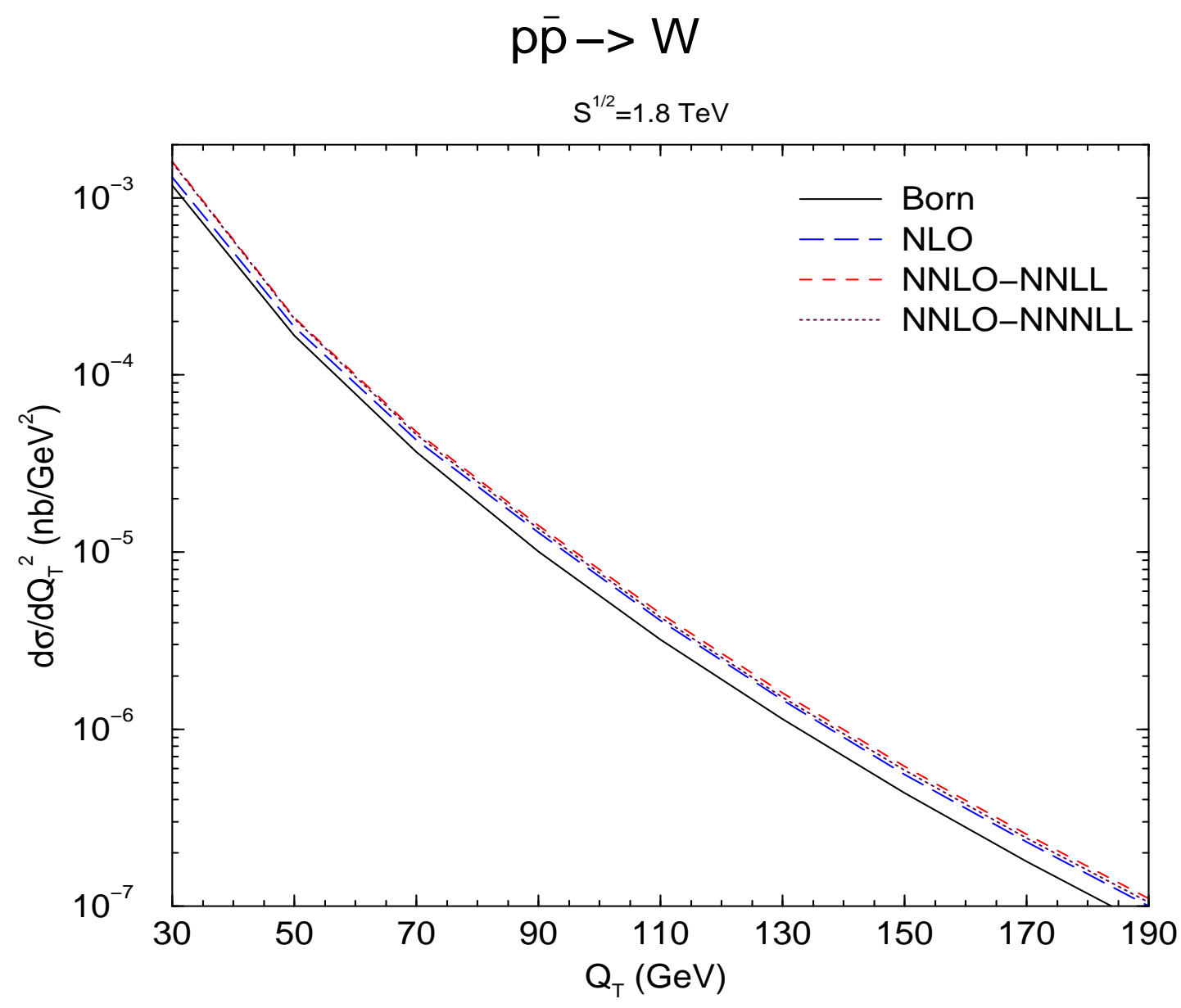

Figure 1: The differential cross section, $d \sigma / d Q_{T}^{2}$, for $W$ hadroproduction in $p \bar{p}$ collisions at the Tevatron with $\sqrt{S}=1.8 \mathrm{TeV}$ and $\mu_{F}=\mu_{R}=Q_{T}$. Shown are the Born (solid line), NLO (long-dashed line), NNLO-NNLL (short-dashed line), and NNLO-NNNLL (dotted line) results.

corrections (which include all the soft and virtual NNLO scale terms) are added. The NNLONNNLL result approaches the scale independence expected of a truly physical cross section.

In Fig. 5 we plot the differential cross section $d \sigma / d Q_{T}^{2}$ at high $Q_{T}$ with $\sqrt{S}=1.8 \mathrm{TeV}$ for two values of scale, $Q_{T} / 2$ and $2 Q_{T}$, often used to display the uncertainty due to scale variation. We note that while the variation of the Born cross section is significant, the variation at NLO is much smaller, and at NNLO-NNNLL it is very small. In fact the two NNLO-NNNLL curves lie on top of the $\mu=Q_{T} / 2$ Born curve, and so does the NLO $\mu=Q_{T} / 2$ curve. These results are consistent with Fig. 4.

In Fig. 6 we plot the transverse momentum distribution, $d \sigma / d Q_{T}^{2}$, for $W$ hadroproduction at the Tevatron Run II with $\sqrt{S}=1.96 \mathrm{TeV}$. Again, we have set $\mu_{F}=\mu_{R}=Q_{T}$. The relative size of the corrections is similar to Fig. 1. In Fig. 7 we display the high- $Q_{T}$ region more clearly. We do not plot the $K$-factors for Run II as they are nearly identical to those for $\sqrt{S}=1.8$ $\mathrm{TeV}$.

In Fig. 8 we plot the differential cross section $d \sigma / d Q_{T}^{2}$ at high $Q_{T}$ with $\sqrt{S}=1.96 \mathrm{TeV}$ for 


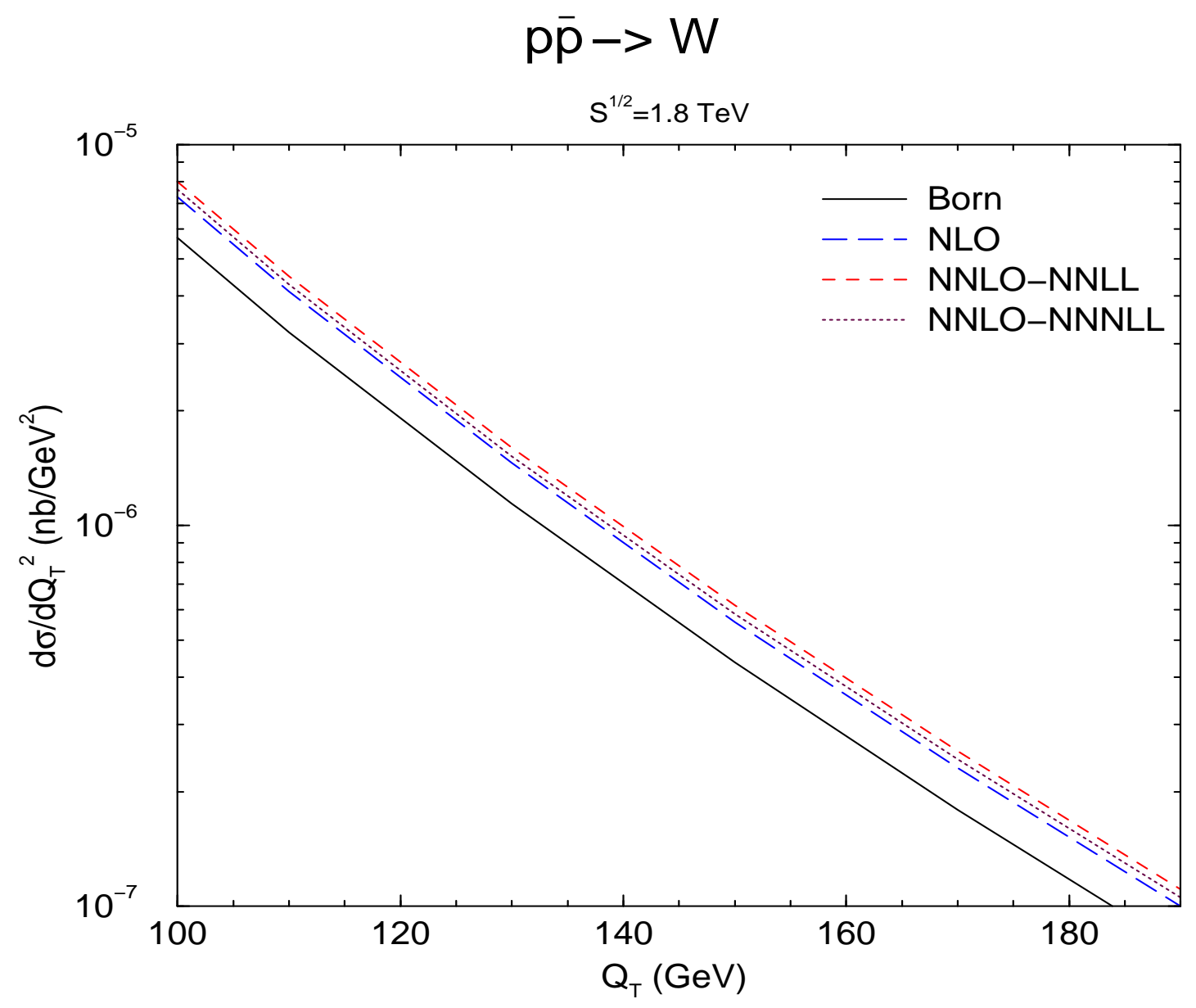

Figure 2: The differential cross section, $d \sigma / d Q_{T}^{2}$, of Fig. 1 at high $Q_{T}$. The labels are the same as in Fig. 1.

two values of scale, $Q_{T} / 2$ and $2 Q_{T}$. The results are analogous to those in Fig. 5.

\section{Conclusion}

We have presented the NNLO soft-gluon corrections for $W$ hadroproduction at large transverse momentum in $p \bar{p}$ collisions, in particular at the Tevatron Run I and II. We have shown that the NLO soft-gluon corrections completely dominate the NLO differential cross section at large transverse momentum, while the NNLO soft-gluon corrections provide modest enhancements and further decrease the factorization and renormalization scale dependence of the transverse momentum distributions. 


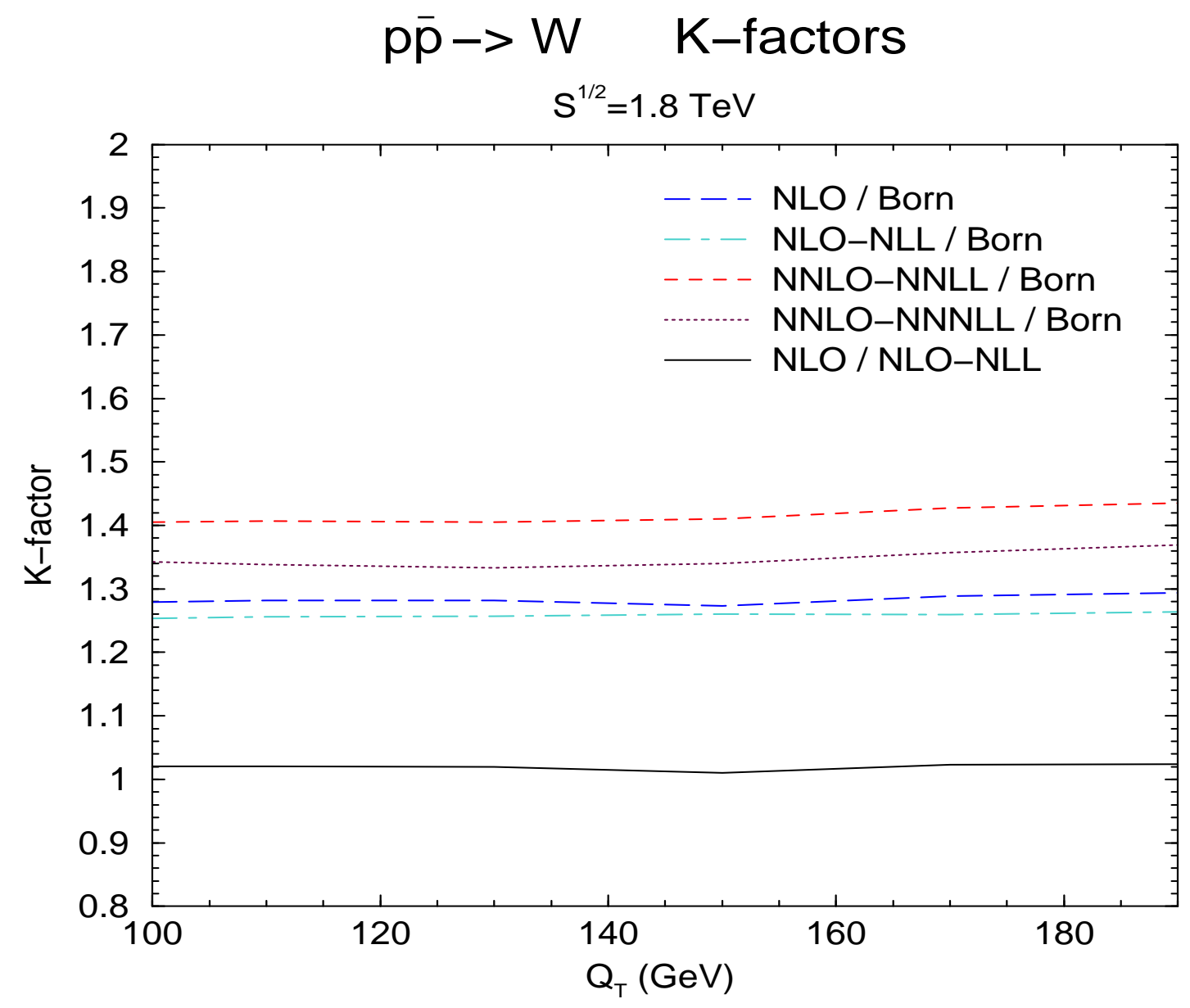

Figure 3: The $K$-factors for the differential cross section, $d \sigma / d Q_{T}^{2}$, for $W$ hadroproduction in $p \bar{p}$ collisions at the Tevatron with $\sqrt{S}=1.8 \mathrm{TeV}$ and $\mu_{F}=\mu_{R}=Q_{T}$. Shown are the $K$ - factors for exact NLO/Born (long-dashed line), NLO-NLL/Born (dash-dotted line), NNLO-NNLL/Born (short-dashed line), and approximate NNLO-NNNLL/Born (dotted line) results. Also shown is the ratio of the exact NLO to the NLO-NLL cross section (solid line).

\section{Acknowledgements}

We are grateful to Richard Gonsalves for providing us with numerical results for the exact NLO corrections. The research of N.K. has been supported by a Marie Curie Fellowship of the European Community programme "Improving Human Research Potential" under contract number HPMF-CT-2001-01221. A.S.V. thanks the II. Institut für Theoretische Physik at the University of Hamburg for hospitality and acknowledges the support of PPARC (Postdoctoral Fellowship: PPA/P/S/1999/00446).

\section{References}

[1] R.K. Ellis and S. Veseli, Phys. Rev. D 60, 011501 (1999). 


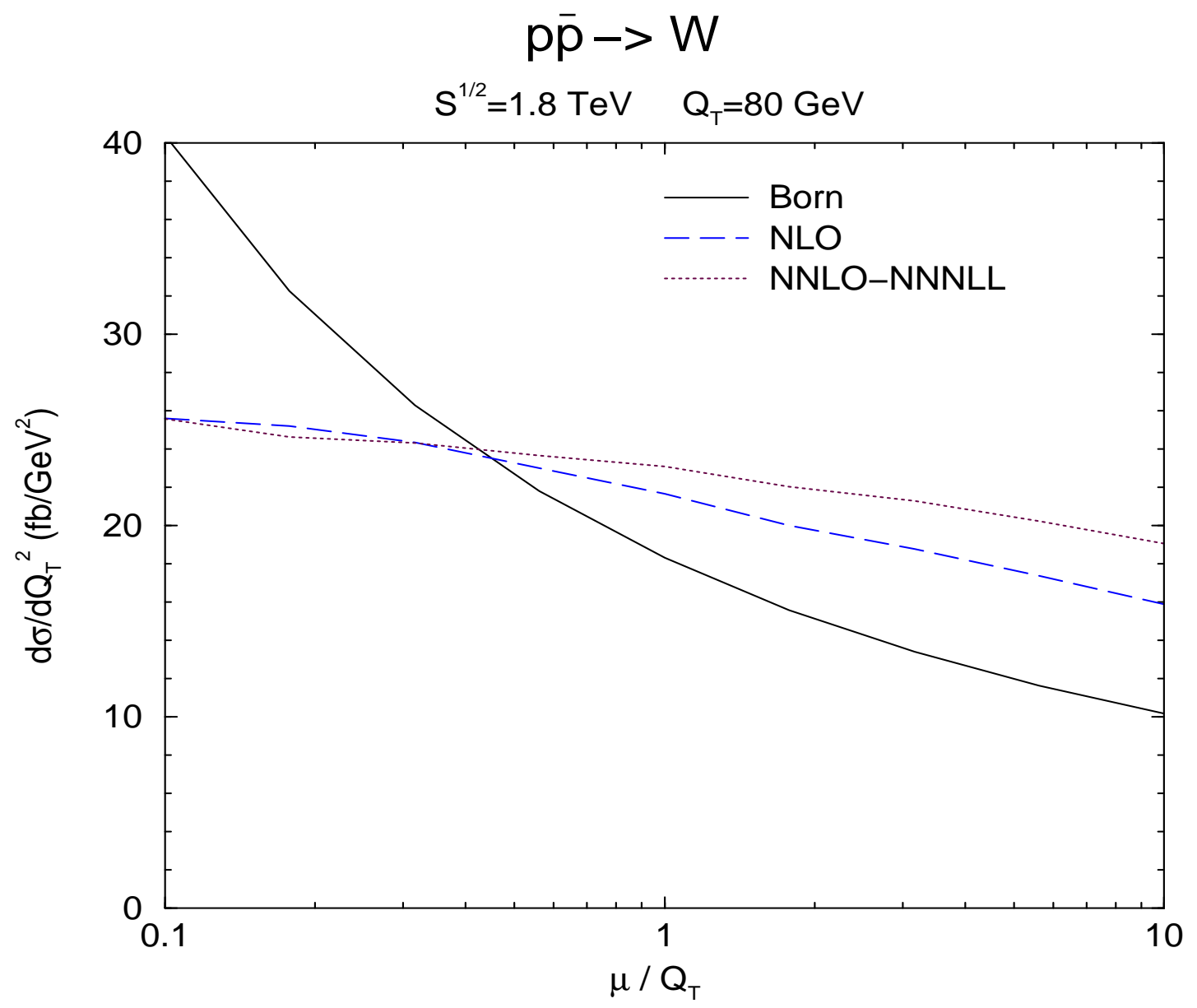

Figure 4: The differential cross section, $d \sigma / d Q_{T}^{2}$, for $W$ hadroproduction in $p \bar{p}$ collisions at the Tevatron with $\sqrt{S}=1.8 \mathrm{TeV}, Q_{T}=80 \mathrm{GeV}$, and $\mu \equiv \mu_{F}=\mu_{R}$. Shown are the Born (solid line), exact NLO (long-dashed line), and NNLO-NNNLL (dotted line) results.

[2] P.B. Arnold and M.H. Reno, Nucl. Phys. B319, 37 (1989); (E) B330, 284 (1990).

[3] R.J. Gonsalves, J. Pawlowski, and C.-F. Wai, Phys. Rev. D 40, 2245 (1989); Phys. Lett. B 252, 663 (1990).

[4] N. Kidonakis and G. Sterman, Phys. Lett. B 387, 867 (1996); Nucl. Phys. B505, 321 (1997).

[5] N. Kidonakis, G. Oderda, and G. Sterman, Nucl. Phys. B525, 299 (1998); B531, 365 (1998).

[6] E. Laenen, G. Oderda, and G. Sterman, Phys. Lett. B 438, 173 (1998).

[7] N. Kidonakis, Int. J. Mod. Phys. A 15, 1245 (2000).

[8] N. Kidonakis and V. Del Duca, Phys. Lett. B 480, 87 (2000); N. Kidonakis, in Proceedings of the EPS-HEP99 Conference, p. 392, hep-ph/9910240. 


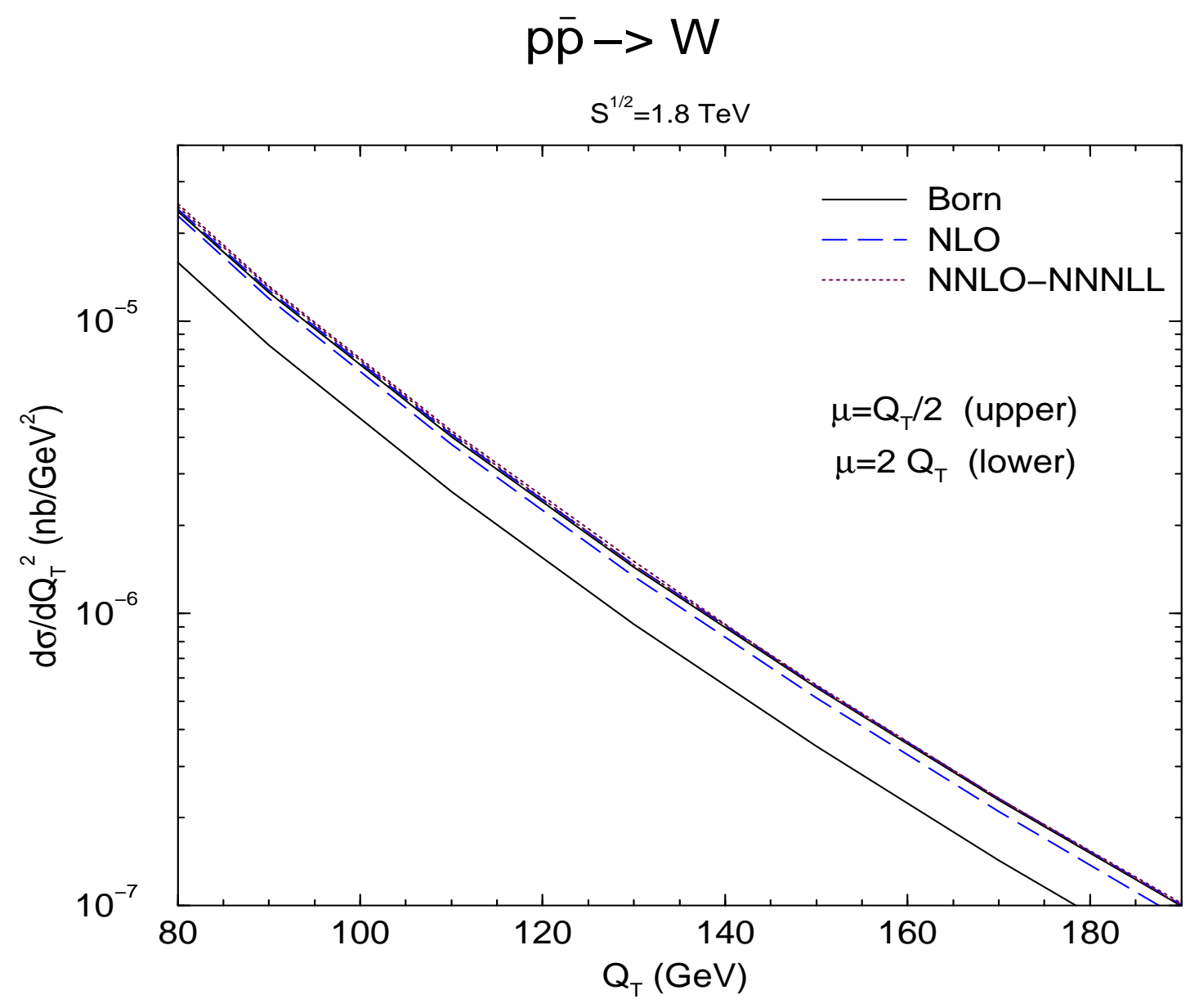

Figure 5: The differential cross section, $d \sigma / d Q_{T}^{2}$, for $W$ hadroproduction in $p \bar{p}$ collisions at the Tevatron with $\sqrt{S}=1.8 \mathrm{TeV}$ and $\mu \equiv \mu_{F}=\mu_{R}=Q_{T} / 2$ or $2 Q_{T}$. Shown are the Born (solid lines), NLO (long-dashed lines), and NNLO-NNNLL (dotted lines) results. The upper lines are with $\mu=Q_{T} / 2$, the lower lines with $\mu=2 Q_{T}$.

[9] N. Kidonakis and J.F. Owens, Phys. Rev. D 61, 094004 (2000); N. Kidonakis, Nucl. Phys. B (Proc. Suppl.) 79, 410 (1999), hep-ph/9905480.

[10] N. Kidonakis and J.F. Owens, hep-ph/0307352, Int. J. Mod. Phys. A (in print).

[11] N. Kidonakis, hep-ph/0303186, Int. J. Mod. Phys. A (in print); in DIS03, hep-ph/0306125, hep-ph/0307207.

[12] R.K. Ellis, D.A. Ross, and S. Veseli, Nucl. Phys. B503, 309 (1997); R.K. Ellis and S. Veseli, Nucl. Phys. B511, 649 (1998).

[13] A. Kulesza and W.J. Stirling, Nucl. Phys. B555, 279 (1999); Eur. Phys. J. C20, 349 (2001).

[14] A. Kulesza, G. Sterman, and W. Vogelsang, Phys. Rev. D 66, 014011 (2002). 


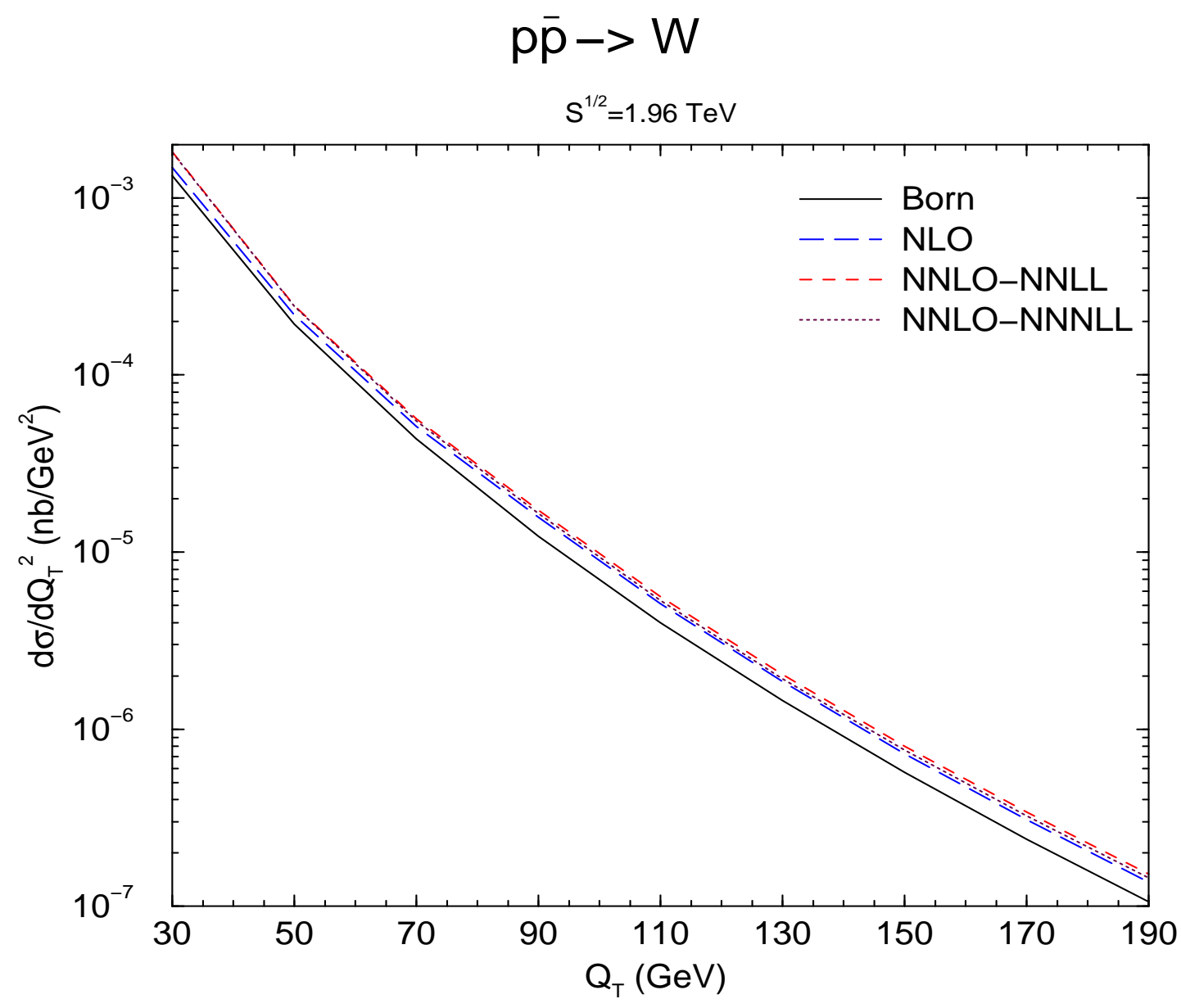

Figure 6: The differential cross section, $d \sigma / d Q_{T}^{2}$, for $W$ hadroproduction in $p \bar{p}$ collisions at the Tevatron Run II with $\sqrt{S}=1.96 \mathrm{TeV}$ and $\mu_{F}=\mu_{R}=Q_{T}$. Shown are the Born (solid line), exact NLO (long-dashed line), NNLO-NNLL (short-dashed line), and NNLO-NNNLL (dotted line) results.

[15] J. Kodaira and L. Trentadue, Phys. Lett. 112B, 66 (1982).

[16] A. Gonzalez-Arroyo, C. Lopez, and F.J. Yndurain, Nucl. Phys. B 153, 161 (1979).

[17] G. Curci, W. Furmanski, and R. Petronzio, Nucl. Phys. B175, 27 (1980); W. Furmanski, and R. Petronzio, Phys. Lett. 97B, 437 (1980).

[18] N. Kidonakis and R. Vogt, hep-ph/0308222, Phys. Rev. D (in print); in EPS-HEP03, hep-ph/0309045.

[19] N. Kidonakis, Phys. Rev. D 64, 014009 (2001); N. Kidonakis, E. Laenen, S. Moch, and R. Vogt, Phys. Rev. D 64, 114001 (2001).

[20] A.D. Martin, R.G. Roberts, W.J. Stirling, and R.S. Thorne, Eur. Phys. J. C 28, 455 (2003). 


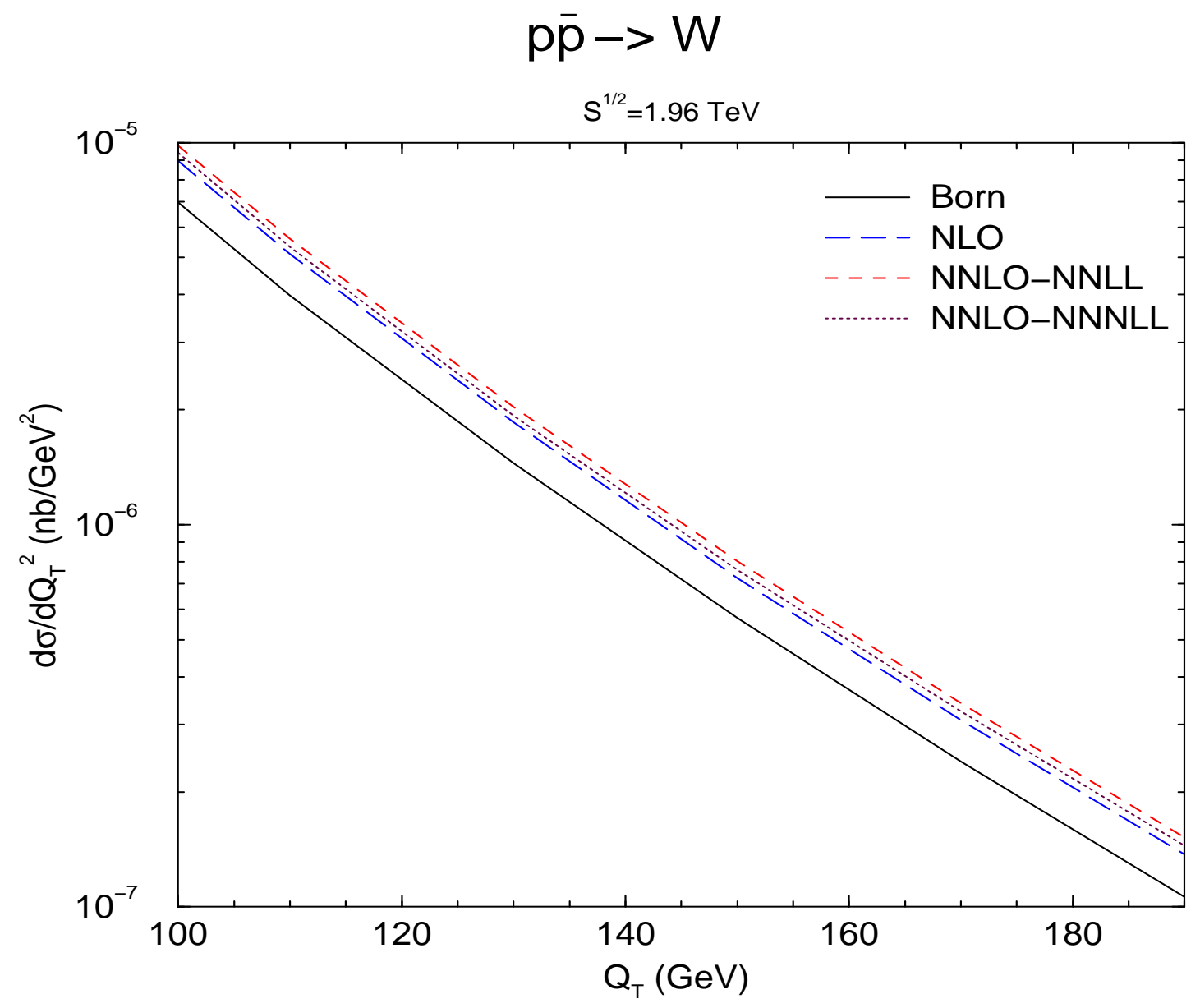

Figure 7: The differential cross section, $d \sigma / d Q_{T}^{2}$, of Fig. 6 at high $Q_{T}$. The labels are the same as in Fig. 6. 


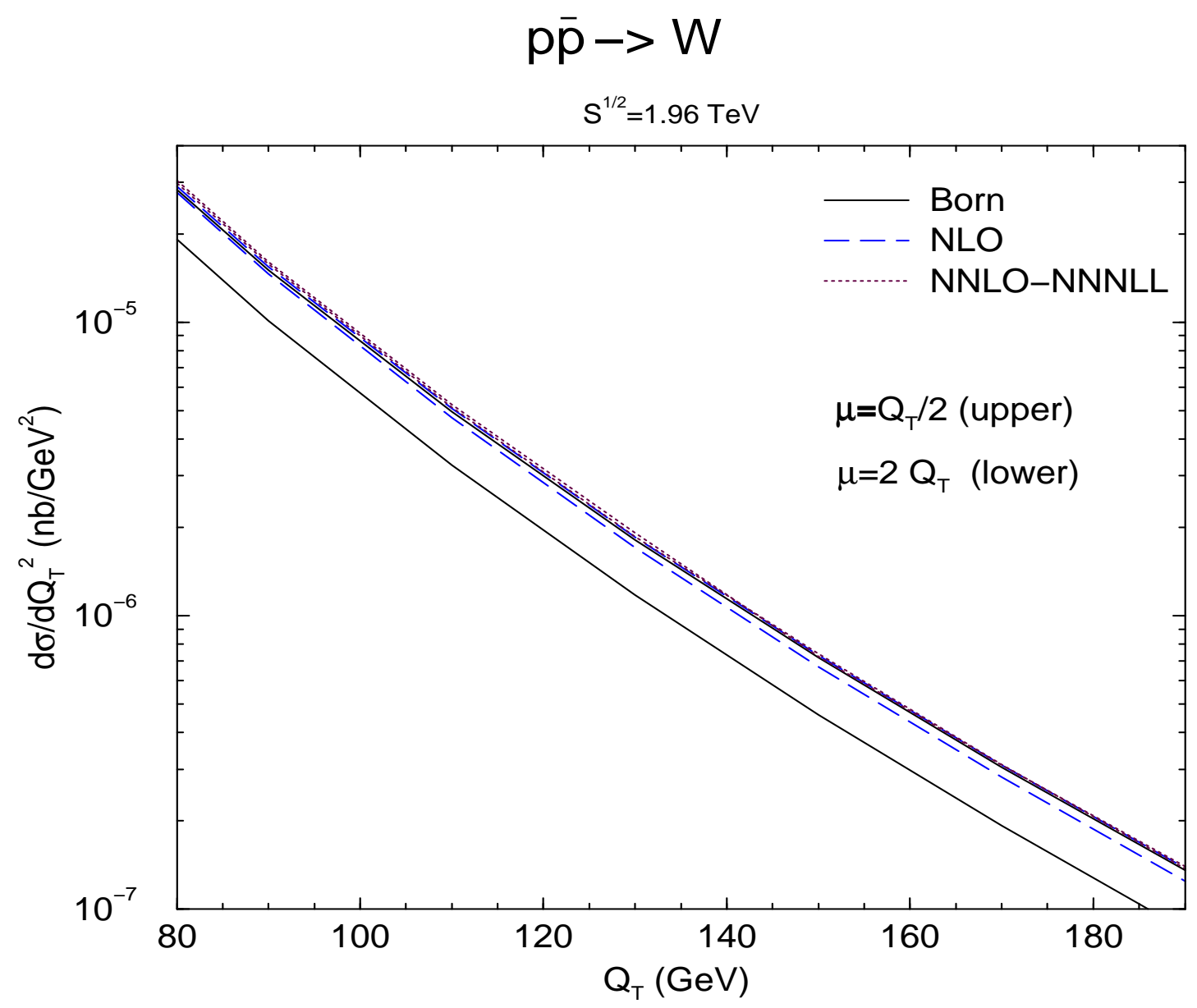

Figure 8: The differential cross section, $d \sigma / d Q_{T}^{2}$, for $W$ hadroproduction in $p \bar{p}$ collisions at the Tevatron with $\sqrt{S}=1.96 \mathrm{TeV}$ and $\mu \equiv \mu_{F}=\mu_{R}=Q_{T} / 2$ or $2 Q_{T}$. Shown are the Born (solid lines), NLO (long-dashed lines), and NNLO-NNNLL (dotted lines) results. The upper lines are with $\mu=Q_{T} / 2$, the lower lines with $\mu=2 Q_{T}$. 